\title{
Coexistence of Neck and Shoulder Disability: Results of a Population-Based Cross-Sectional Study on Normative Scores and Multifactorial Risk Factors for Neck and Shoulder Problems
}

\author{
Juliane Koller ${ }^{1,2}$, Carsten Bismarck ${ }^{1}$, Sona $\operatorname{Krebs}^{1}$, Wolfgang Hitzl ${ }^{3}$, Michael Mayer ${ }^{2,4}$, Heiko Koller ${ }^{4,5}$ \\ ${ }^{1}$ Department of General Surgery and Traumatology, Hospital zum Heiligen Geist Fritzlar, Fritzlar, Germany \\ ${ }^{2}$ Center for Spine and Scoliosis Surgery, Schoen-Klinik Vogtareuth, Vogtareuth, Germany \\ ${ }^{3}$ Research Office for Biostatistics, Paracelsus Medical University Salzburg, Salzburg, Austria \\ ${ }^{4}$ Department for Orthopedic Surgery and Traumatology, Paracelsus Medical University Salzburg, Salzburg, Austria \\ ${ }^{5}$ Department of Neurosurgery, Klinikum Rechts der Isar, Technical University of Munich, München, Germany
}

\section{Study Design: Cross-sectional population-based study.}

Purpose: The study objective was to evaluate the coexistence of neck- and shoulder-disability, to establish normative scores for Neck Disability Index (NDI), Visual Analog Scale (VAS)-neck, VAS-arm, Quick Disability of Shoulder and Hand (Quick-DASH), and modified Constant score (mConstant score), and to determine the influence of psychological distress (Hospital Anxiety and Depression Scale [HADS]) on the disability measures. The authors also investigated the distribution of dysphagia across the population and its relation to the NDI scores.

Overview of Literature: Several factors can adversely influence the clinical outcomes after cervical surgeries. The interaction of neck and shoulder disability in the perspective of psychological distress is not well understood.

Methods: Prospective questionnaire-based assessment was performed for 1,000 participants. Questionnaires consisted of validated generic and disease-specific queries and specific questions. The survey included patients without pathologies of cervical spine/shoulders/upper extremities.

Results: Mean age of participants was 39 years. The average neck VAS score was 1.2, NDI\% was 7.3, arm VAS score was 0.8, QuickDASH was 6.2, mConstant score was 70.7, HADS-A score was 4.9, and HADS-D score was 3.2. The psychological scores showed a significant correlation with neck- and shoulder-disability $(p<0.0001, r=0.3$ to $r=0.5)$. However, correlations between neck (NDI\%, neck VAS score) and shoulder disability (mConstant score, arm VAS score, Quick-DASH) were stronger ( $p<0.0001, r=0.5$ to $r=0.6)$. A body mass index $>35 \mathrm{~kg} / \mathrm{m}^{2}$ influenced shoulder-disability $(p<0.005)$ and psychological distress (HADS-D score, $\left.p<0.00001\right)$. Limited neck rotation was present in those with higher age, psychological distress, neck and shoulder disability $(p<0.001)$.

Conclusions: Normative scores for neck and shoulder disability were established. The outcomes of cervical spine surgery can be normalized to these results. A better understanding of the interdependencies of neck and shoulder disability and psychological distress would enable superior decision-making and patient counseling.

Keywords: Cervical; Spine; Neck Disability Index; Normal scores; Dysphagia; Depression

Received Dec 27, 2019; Revised Mar 22, 2020; Accepted Mar 24, 2020

Corresponding author: Heiko Koller

Department of Neurosurgery, Klinikum Rechts der Isar, Technical University Munich, Ismaninger Str. 22, 81675 Munich, Germany

Tel: +49-16094609732, E-mail: heiko.koller@t-online.de 


\section{Introduction}

Neck pain is one of the four most commonly reported musculoskeletal disorders that increases the health care cost. The incidence of chronic non-specific neck pain is reported in about 15\% women and 9\% men. The following risk factors for non-specific neck pain have been identified: workplace bullying, sleeping problems, body mass index (BMI), female sex, older age, high job demands, low social/work support, tobacco use, and a history of low back disorder [1-5]. Other researches have reported a significant association among distress, anxiety, and neck pain $[4,6]$. It is noteworthy that a literature review concluded that previous studies on risk factors provide limited evidence; therefore, there is insufficient research on the predictive nature of various variables [2]. Regarding post-surgery surgical neck pain, the Neck Disability Index (NDI) is proven as the most important factor in explaining the short- and long-term surgical outcomes [7], while the influence of other variables remains unclear. In a similar manner, outcome studies have shown that postoperative patient satisfaction mainly depends on postoperative pain relief.

The NDI is an established outcome measure [8]. However, there is lack of access to large NDI databases that would enable matching of outcomes in patients receiving cervical spine treatment with a group of normal healthy subjects. This would be valuable and put surgical results in perspective of a population-based normative distribution of neck disability.

Similar to that with perioperative neck pain, dysphagia is a significant parameter that influences perioperative outcome and complication rates in cervical spine surgery. It has been reported in 11\%-64\% subjects after anterior cervical surgery and even in $13 \%$ of patients following posterior cervical surgery. Risk factors for postoperative dysphagia have been identified [9]; however, there is lack of normative data suitable for benchmarking surgical outcomes.

The objectives of our current study were twofold. The first goal was to establish normative scores for the measures of neck and shoulder disability (NDI\%, neck Visual Analog Scale [VAS] score, arm VAS score, Quick Disability of Shoulder and Hand [Quick-DASH], modified Constant score [mConstant score]) as well as the distribution and severity of dysphagia in a large cohort. The second goal was to improve the understanding of coexisting neck and shoulder disability and identify predictors for increased neck and shoulder disability. We sought to determine the influence of psychological distress (Hospital Anxiety and Depression Scale [HADS]) and to identify risk factors (e.g., smoking, BMI, age) for elevated disability.

\section{Materials and Methods}

IRB approval was obtained from Department of General Surgery and Traumatology, Hospital zum Heiligen Geist Fritzlar, Germany, and patients provided informed consent for the publication of de-identified data. Prospective cross-sectional assessment of normative scores for neck and shoulder disability and dysphagia in normal individuals until completion of 1,000 eligible surveys. In order to enroll consecutive 1,432 individuals to meet the target sample size, institutional personnel, patients presenting for minor medical requests to the outpatient clinic of a primary care center, as well as accompanying persons were asked to complete a survey set. Participants were included and defined as normal individuals if they had no history of cervical spine, neck, shoulder, or upper extremity disorders or injuries and if they were not seeking consultation for cervical or shoulder/arm referred pain and disability. Further inclusion criteria were age 16-90 years, Caucasian origin, ability to understand county language, and ability to complete the survey. The survey consisted of validated generic and disease-specific questions and a modification of the Constant score (NDI\%, neck VAS score, shoulder VAS score, arm VAS score, Quick-DASH, HADS score, mConstant score).

\section{Disease-specific scores}

The NDI is a validated outcome measure used for assessing neck pain and function [10]. The index ranges from $0 \%-100 \%$, with higher scores indicating increased disability. The NDI can be graded into the following five disability groups: $0 \%-20 \%$ shows no/minimal disability, $21 \%-40 \%$ shows moderate disability, $41 \%-60 \%$ shows severe disability, $61 \%-80 \%$ indicates crippling, $81 \%-100 \%$ indicates inability to get out of bed. Any NDI $>20 \%$ was considered to indicate significant impairment.

Pain intensity in neck and/or arm was assessed using the visual analogue scale, and subjects were asked to rate the pre-assessment period of 4 weeks. Range was $0-10$, 
with higher values indicating more pain. Significant arm or neck pain was defined as a VAS score $\geq 5$, respectively. For statistical analysis, patients with any VAS $\geq 5$ (arm or neck) were identified.

The Quick-DASH is a validated shoulder specific functional outcome measure consisting of 11 questions [11]. The range is $0-100$ points, with higher scores indicating increased disability. In a subdomain DASH-work, results for work related shoulder functions are summarized.

The Constant-Murley score is a physician-assessed questionnaire. For the current study, modifications were applied to allow patient assessment as per the survey parameters. A previous study has shown a high correlation between physician-assessed and patient-based versions of the Constant score [12]. Pain levels were assessed using a linear scale as well as single questions; the inhibitions of daily living were recorded equal to the Constant score. The range of movement was assessed by ticking off pictures with the appropriate arm position demonstrated in increasing angulations and awarded with points $(0-10)$. The individual scores were divided through the maximum of 90 points that could be reached. Function grading was as follows: $>30$, poor; $21-30$, fair; $11-20$, good; $<11$, excellent). The sum of shoulder range of motion $\left(\mathrm{ROM},{ }^{\circ}\right)$ was assessed as a continuous variable for the statistical analysis.

\section{Psychological scores}

The HADS is a validated and reliable self-rating scale that measures anxiety and can assess the symptom severity and caseness of anxiety disorders (HADS-A) and depression (HADS-D) [13]. The questionnaire comprises 14 questions. Scores range from $0-21$ points and can be categorized as follows: normal (0-7, group A), mild (8-10, group B), moderate (11-14, group C), and severe (15-21, group D) psychological distress. For statistical purposes, mild, moderate, and severe HADS-A or HADS-D groups were merged into a group with a significant HADS score (>7 points).

\section{Generic scores and questions}

Patients were asked whether they had experienced any dysphagia in the previous 4 weeks. Patient-reported dysphagia was assessed according to the 4-point grading system proposed by Bazaz et al. [14] for liquid and solid foods. Further questions were regarding activities of daily living (AODL), social activities, working life, sleep disorders, occupational status, health care utilization, and duration of sensory dysfunction (numbness) in the upper and lower extremities during the previous 4 weeks. ROM of the cervical spine was assessed by ticking off pictures revealing increasingly angulated positions of neck rotation.

\section{Statistical methods}

Data consistency was checked and screened for outliers as well as normality by using quartile plots. Continuous variables were also tested for normality using the Kolmogorov-Smirnov test. Cross-tabulation tables were computed and analyzed using Fisher's exact test or Pearson's chi-square test. Independent and dependent Student $t$-tests were used to test continuously distributed variables when assumptions were fulfilled. Pearson's and Spearman correlation coefficients were computed and tested. One factorial analysis of variance (ANOVA), repeated ANOVA models, and corresponding least significant difference tests for pairwise comparisons of means were performed; 95\% confidence intervals (CIs) for the means were computed and illustrated using whisker plots. All the reported tests were two-sided. All $p$-values $<0.05$ were considered statistically significant.

A forward stepwise algorithm for variable selection was applied. A statistical model was built in a learning sample $(\mathrm{n}=753)$ and tested in an independent cross-validation sample ( $n=247)$. In order to rank the variables, Wald's test statistic was used to select the significant predictors. Further, $5 \%$ and $95 \%$ quartiles for raw residuals were computed in the learning and cross-validation sample to assess the stability and generalizability of the models. The crossvalidation sample was established based on computergenerated random numbers. For dichotomous outcomes, general linear discriminant analyses were applied, evaluated in the learning sample, and tested in the independent cross-validation sample by using classification matrices. Again, a forward stepwise variable algorithm was applied, and the coefficients of the canonical correlation functions were tested based on the Wilk's lambda test statistic. Receiver operation characteristic (ROC) curves were computed and tested to assess the discriminant power of the individual. For this, area under curve (AUC) with 95\% CI was computed, and non-inferiority tests were applied. A 
$p$-value $<5 \%$ indicated a significant difference. All analyses were performed using Statistica ver. 10.0 (StatSoft Inc., Tulsa, OX, USA; 2011) and Mathematica ver. 7.0 (Wolfram Research Inc., Champaign, IL, USA; 2008).

\section{Results}

\section{Patient population}

The study population included 525 men and 475 women; $34 \%$ of the participants were smokers, the average patient age was 39 years (range, 16-90 years; 95\% CI, 25.7-26.3

Table 1. Results of validated disease and generic outcome measures

\begin{tabular}{|lcr|}
\hline Variable & $\begin{array}{c}\text { Mean } \pm 1 \\
\text { standard } \\
\text { deviation }\end{array}$ & $\begin{array}{c}95 \% \\
\text { Confidence } \\
\text { interval }\end{array}$ \\
\hline Neck disability & $1.17 \pm 1.76$ & $1.06-1.28$ \\
\hline Visual Analog Scale-neck & $7.25 \pm 9.26$ & $6.67-7.82$ \\
\hline Neck Disability Index & & \\
\hline Shoulder-disability & $70.65 \pm 8.25$ & $70.14-71.17$ \\
\hline Modified Constant score & $6.21 \pm 11.37$ & $5.51-6.92$ \\
\hline Ouick Disability of Shoulder and Hand & $38.56 \pm 4.20$ & $38.30-38.82$ \\
\hline Shoulder-range of motion $\left(^{\circ}\right)$ & & \\
\hline Psychological distress & & $4.71-5.17$ \\
\hline HADS-anxiety disorders & $3.94 \pm 3.69$ & $2.97-3.41$ \\
\hline HADS-depression & $3.19 \pm 3.53$ & \\
\hline
\end{tabular}

HADS, Hospital Anxiety and Depression Scale.

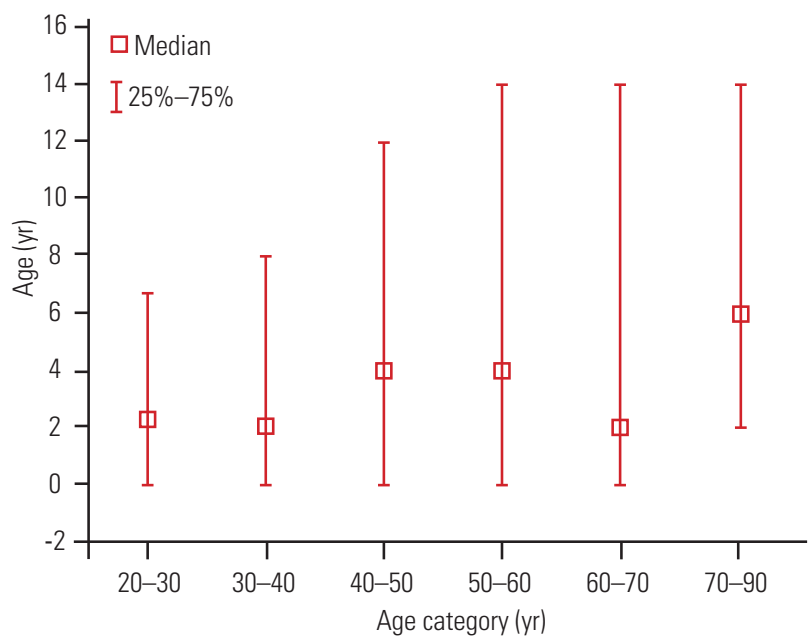

Fig. 1. Age dependent distribution of Neck Disability Index (\%) in 1,000 individuals. years), and mean BMI was $26 \pm 5 \mathrm{~kg} / \mathrm{m}^{2}$ (95\% CI, 37.9-39.9 $\left.\mathrm{kg} / \mathrm{m}^{2}\right)$. The normative values for the validated outcome measures, stratified into neck disability, shoulder-disability, and psychological distress are summarized in Table 1. Distribution of NDI according to age is illustrated in Fig. 1.

The NDI $>20 \%$ was considered to indicate significant impairment. The threshold selected was higher than the mean NDI of 7.25 ( \pm 1 standard deviation, 9.26). Significant NDI showed an age-dependent distribution $(p<0.0001)$ with an average prevalence of $11 \%$ (Fig. 2). In subjects aged $20-30$ years, only $2 \%$ had a NDI $>20 \%$, increasing with age up to 50-60 years; subjects aged 50-60 years had the highest prevalence of NDI $>20 \%(18 \%)$. In the age group of $60-70$ years, NDI $>20 \%$ decreased to $14 \%$ of participants. The intergroup differences were significant $(p<0.0001)$.
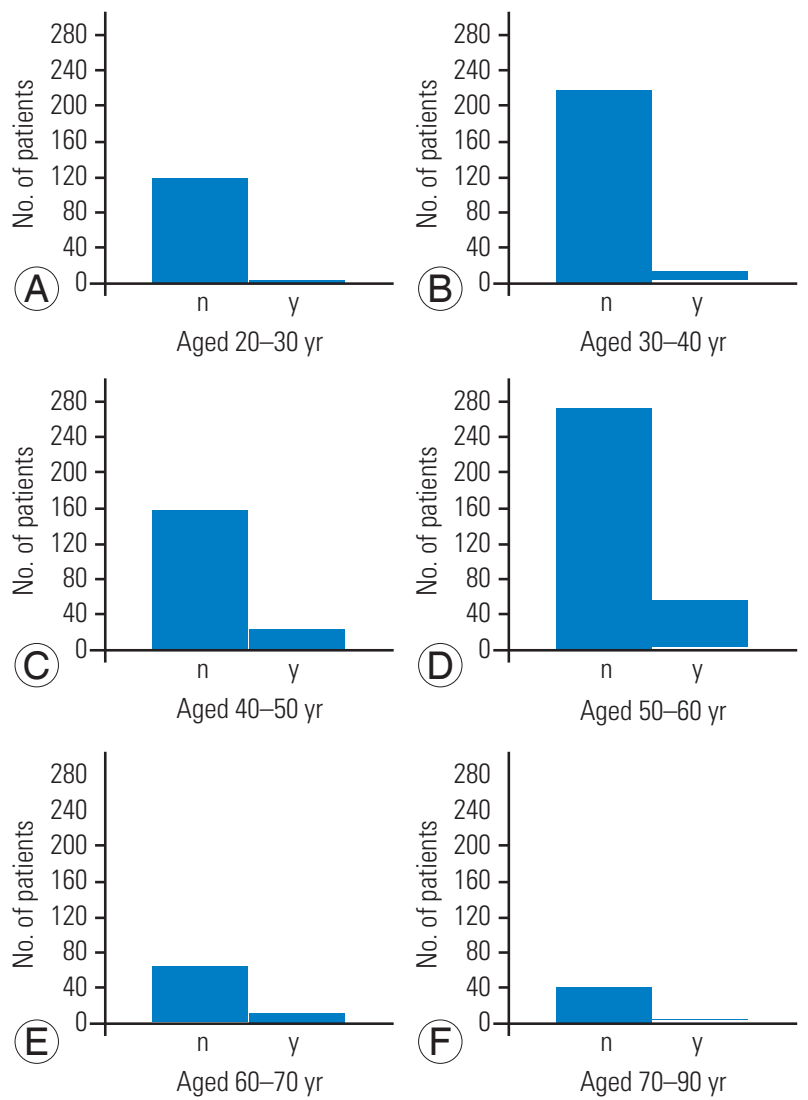

Fig. 2. Age dependent distribution of a significant NDI, defined as NDI $>20 \%$. $\mathrm{Y}$-axis denotes number of patients; $\mathrm{x}$-axis denotes age categories. (A) Category: aged 20-30 years; (B) category: aged 30-40 years; (C) category: aged 40-50 years; (D) category: aged 50-60 years; (E) category: aged 60-70 years; and (F) category: aged 70-90 years. NDI, Neck Disability Index; y, yes; n, no. 


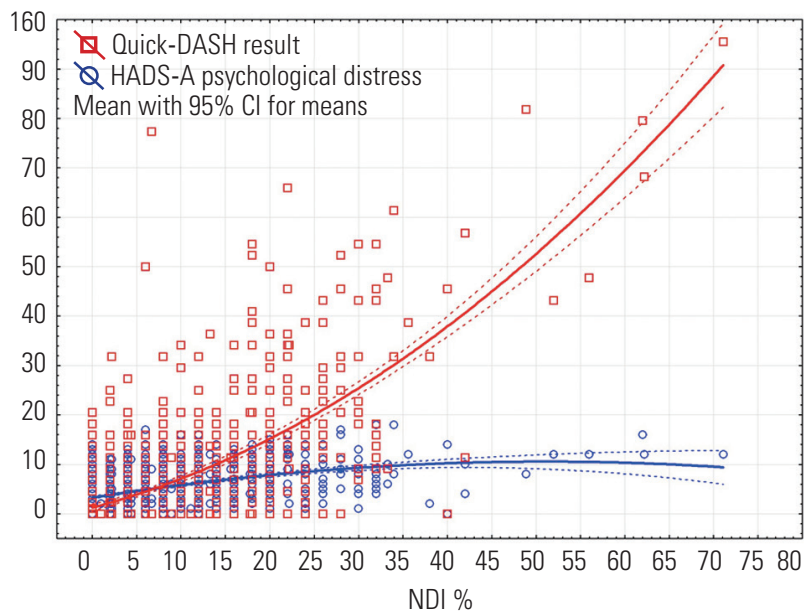

Fig. 3. Correlation analysis of NDI (\%) with Quick-DASH results $(r=0.6$, $p<0.000001)$ as well as NDI (\%) and HADS-A $(r=0.5, p<0.000001)$. NDI, Neck Disability Index; Quick-DASH, Quick Disability of Shoulder and Hand; HADS-A Hospital Anxiety and Depression Scale-anxiety disorders; $\mathrm{Cl}$, confidence interval.

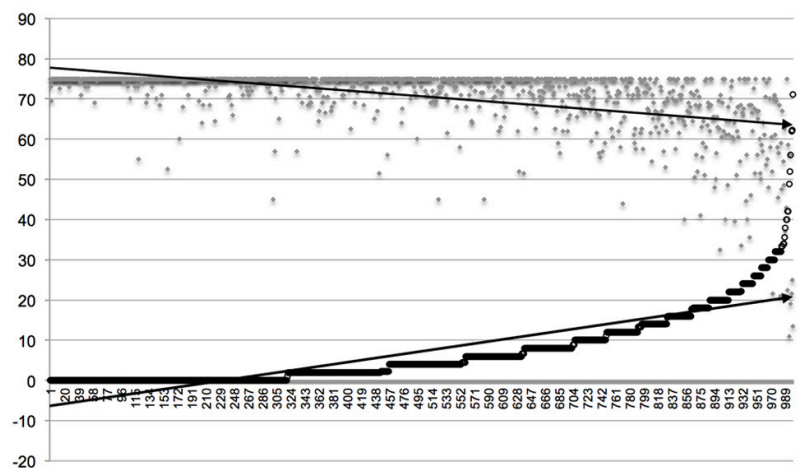

Fig. 4. Correlation analysis of NDI (\%) (indicated by $\bigcirc$ ) with mConstant score (indicated by $\diamond$ ); $r=0.6, p<0.000001$. Y-axis denotes NDI (\%)/Constant score (points) and $\mathrm{x}$-axis denotes number of patients $(\mathrm{n}=1,000)$. Arrow lines indicate extrapolated trend lines. NDI, Neck Disability Index; mConstant score, modified Constant score.

Table 2. Results of statistical correlation analysis between indicators of neck disability (NDI, VAS-neck), shoulder-disability (Quick-DASH, VAS-arm, mConstant score), psychological distress (HADS-A, HADS-D), and patient characteristics (age, BMI)

\begin{tabular}{|c|c|c|c|c|c|c|c|c|c|c|c|}
\hline Variable & Category & VAS-neck & NPDI & VAS-arm & $\begin{array}{l}\text { mConstant } \\
\text { score }\end{array}$ & $\begin{array}{l}\text { Quick- } \\
\text { DASH }\end{array}$ & $\begin{array}{c}\text { Shoulder- } \\
\text { ROM }\end{array}$ & HADS-A & HADS-D & Age & $\mathrm{BMI}$ \\
\hline \multirow[t]{2}{*}{ VAS-neck } & $r$ & 1 & 0.76 & 0.58 & -0.58 & 0.55 & -0.34 & 0.41 & 0.34 & 0.11 & 0.06 \\
\hline & $p$-value & & $<0.0001$ & $<0.0001$ & $<0.0001$ & $<0.0001$ & $<0.0001$ & $<0.0001$ & $<0.0001$ & $<0.0001$ & 0.048 \\
\hline \multirow[t]{2}{*}{ NPDI } & $r$ & 0.76 & 1 & 0.52 & -0.58 & 0.58 & -0.35 & 0.49 & 0.42 & 0.10 & 0.05 \\
\hline & $p$-value & $<0.0001$ & & $<0.0001$ & $<0.0001$ & $<0.0001$ & $<0.0001$ & $<0.0001$ & $<0.0001$ & $<0.0001$ & 0.13 \\
\hline \multirow[t]{2}{*}{ VAS-arm } & $r$ & 0.58 & 0.52 & 1 & -0.58 & 0.53 & -0.33 & 0.35 & 0.34 & 0.19 & 0.09 \\
\hline & $p$-value & $<0.0001$ & $<0.0001$ & & $<0.0001$ & $<0.0001$ & $<0.0001$ & $<0.00001$ & $<0.0001$ & $<0.0001$ & 0.004 \\
\hline \multirow[t]{2}{*}{ mConstant score } & $r$ & -0.58 & -0.58 & -0.58 & 1 & -0.71 & 0.69 & -0.39 & -0.38 & -0.33 & -0.15 \\
\hline & $p$-value & $<0.0001$ & $<0.0001$ & $<0.0001$ & & $<0.0001$ & $<0.0001$ & $<0.0001$ & $<0.0001$ & $<0.0001$ & $<0.0001$ \\
\hline \multirow[t]{2}{*}{ Quick-DASH } & $r$ & 0.55 & 0.58 & 0.53 & -0.71 & 1 & -0.48 & 0.43 & 0.41 & 0.37 & 0.11 \\
\hline & $p$-value & $<0.0001$ & $<0.0001$ & $<0.0001$ & $<0.0001$ & & $<0.0001$ & $<0.0001$ & $<0.0001$ & $<0.0001$ & $<0.001$ \\
\hline \multirow[t]{2}{*}{ Shoulder-ROM } & $r$ & -0.34 & -0.35 & -0.33 & 0.69 & -0.48 & 1 & -0.19 & -0.24 & -0.35 & -0.22 \\
\hline & $p$-value & $<0.0001$ & $<0.0001$ & $<0.0001$ & $<0.0001$ & $<0.0001$ & & $<0.0001$ & $<0.0001$ & $<0.0001$ & $<0.0001$ \\
\hline \multirow[t]{2}{*}{ HADS-A } & $r$ & 0.41 & 0.49 & 0.35 & -0.39 & 0.43 & -0.19 & 1 & 0.66 & 0.09 & 0.05 \\
\hline & $p$-value & $<0.0001$ & $<0.0001$ & $<0.00001$ & $<0.0001$ & $<0.0001$ & $<0.0001$ & & $<0.0001$ & 0.005 & 0.096 \\
\hline \multirow[t]{2}{*}{ HADS-D } & $r$ & 0.34 & 0.42 & 0.34 & -0.38 & 0.41 & -0.24 & 0.66 & 1 & 0.24 & 0.16 \\
\hline & $p$-value & $<0.0001$ & $<0.0001$ & $<0.0001$ & $<0.0001$ & $<0.0001$ & $<0.0001$ & $<0.0001$ & & $<0.0001$ & $<0.0001$ \\
\hline \multirow[t]{2}{*}{ Age } & $r$ & 0.11 & 0.10 & 0.19 & -0.33 & 0.37 & -0.35 & 0.09 & 0.24 & 1 & 0.33 \\
\hline & $p$-value & $<0.0001$ & $<0.0001$ & $<0.0001$ & $<0.0001$ & $<0.0001$ & $<0.0001$ & 0.005 & $<0.0001$ & & $<0.0001$ \\
\hline \multirow[t]{2}{*}{ BMI } & $r$ & 0.06 & 0.05 & 0.09 & -0.15 & 0.11 & -0.22 & 0.05 & 0.16 & 0.33 & 1 \\
\hline & $p$-value & 0.048 & 0.13 & 0.004 & $<0.0001$ & $<0.001$ & $<0.0001$ & 0.096 & $<0.0001$ & $<0.0001$ & \\
\hline
\end{tabular}

Bold-typed cells indicate correlation strength $R>0.4$.

NDI, Neck Disability Index; VAS, Visual Analog Scale; Quick-DASH, Quick Disability of Shoulder and Hand; mConstant score, modified Constant score; HADS-A, Hospital Anxiety and Depression Scale-anxiety disorders; HADS-D, Hospital Anxiety and Depression Scale-depression; BMI, body mass index; NPDI, Neck Pain Disability Index; ROM, range of motion; $r$, Spearman correlation coefficient. 
Table 3. Results of generalized linear model for discrimination strength of continuous variables for Neck Disability Index (\%)

\begin{tabular}{lccccc} 
Variable & Estimate & Standard deviation & Wald's test statistic & 95\% Confidence index & $p$-value \\
HADS-A & 0.051 & 0.009 & 35.290 & 0.034 to 0.068 & $<.000001$ \\
Quick-DASH & 0.012 & 0.002 & 21.582 & 0.007 to 0.017 & 0.000003 \\
\hline mConstant score & -0.028 & 0.008 & 12.720 & -0.044 to -0.013 & 0.0004 \\
VAS-arm & 0.050 & 0.016 & 9.330 & 0.018 to 0.082 & 0.002 \\
\hline Age & -0.003 & 0.002 & 2.567 & -0.007 to 0.001 & 0.11 \\
HADS-D & 0.002 & 0.008 & 0.045 & -0.014 to 0.018 & 0.83 \\
\hline BMI & 0.000 & 0.005 & 0.000 & -0.010 to 0.010 & 0.99 \\
\hline
\end{tabular}

HADS-A, Hospital Anxiety and Depression Scale-anxiety disorders; Quick-DASH, Quick Disability of Shoulder and Hand; mConstant score, modified Constant score; VAS, Visual Analog Scale; HADS-D, Hospital Anxiety and Depression Scale-depression; BMI, body mass index.

\section{Association between psychological distress and disability}

Psychological distress scores (HADS scores) showed significant correlation with neck and shoulder disability $(p<0.05$, $r=0.3$ to $r=0.5$ ). The correlations between neck (NDI\%, neck VAS score) and shoulder disability (mConstant score, arm VAS score, Quick-DASH) were stronger $(p<0.0001$, $r=0.5$ to $r=0.6$ ). The main correlations between neck and shoulder disability as well as psychological distress measures are illustrated in Figs. 3 and 4 and in Table 2.

To study the main parameters that influence the NDI (\%), multivariate analyses including continuous and discrete variables were performed. The generalized linear model suggested that in descending order, HADS-A score had largest discrimination power for NDI (\%) followed by that for mConstant score, sum of shoulder-ROM, QuickDASH, patient age, HADS-D score, and BMI. The results are summarized in Table 3; 5\% and 95\% quartiles of the residuals were 7.4 and 13.4 in the learning sample and -6.8 and 12.2 in the cross-validation sample, respectively, indicating good model performance and showing that the results are stable in the learning and test samples. Regarding the prediction of a significant NDI (\%), the general linear discriminant analysis showed that for the category "significant NDI", the parameters with largest discrimination power were Quick-DASH (Wilk's lambda $=0.953$, $p<0.000001$ ), HADS-A score (Wilk's lambda $=0.980$, $p=0.00009$ ), mConstant score (Wilk's lambda $=0.983$, $p=0.0004$ ), and arm VAS score (Wilk's lambda $=0.991$, $p=0.01$ ) in descending order. The total correct classification rate was $91 \%$ in the learning sample and $93 \%$ in the cross-validation sample, indicating that the model performs well and the results are stable in the learning and test samples.

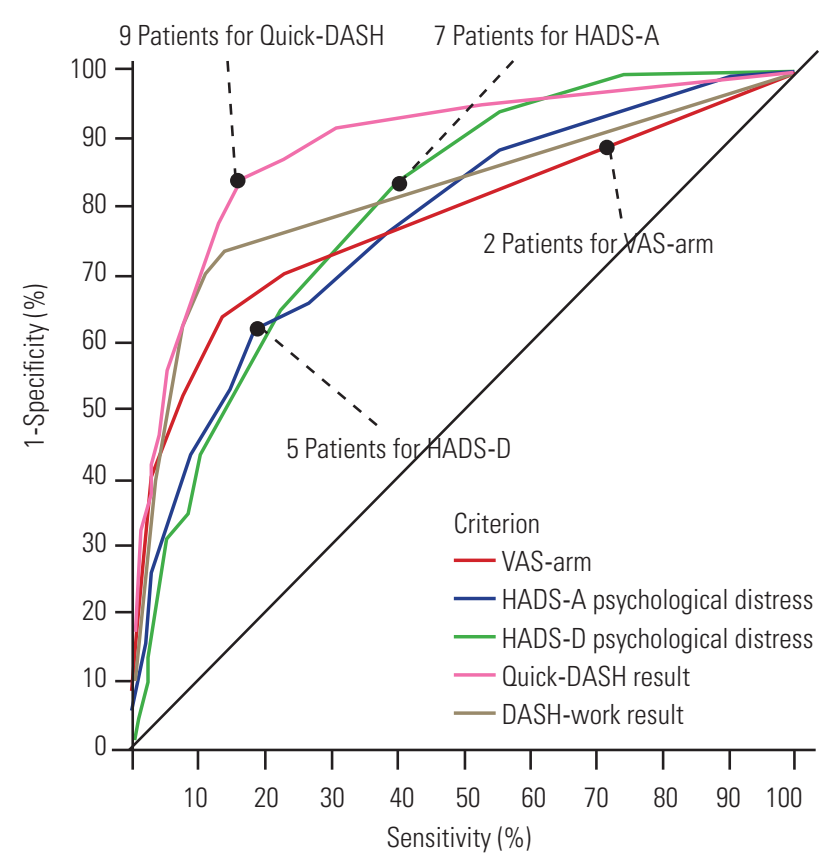

Fig. 5. Receiver operation characteristic calculations and curves for prediction of 'significant NDI (\%)' with established cut-off points for shoulder-disability and psychological distress variables and related sensitivity and specificity data. For example, for prediction of 'significant NDI' a cut-off at 2 points for the VASarm could be established with a sensitivity of $60 \%$ and a specificity of $88 \%$; a cut-off at 7 points for the HADS-A with a sensitivity of $66 \%$ and a specificity of $74 \%$; a cut-off at 5 points for the HADS-D with a sensitivity of $66 \%$ and a specificity of $77 \%$; a cut-off at 9.1 points for the Quick-DASH with a sensitivity of $84 \%$ and a specificity of $83 \%$. NDI, Neck Disability Index; VAS, Visual Analog Scale; HADS-A, Hospital Anxiety and Depression Scale-anxiety disorders; HADS-D, Hospital Anxiety and Depression Scale-depression; Quick-DASH, Quick Disability of Shoulder and Hand.

To identify more precise predictors of NDI $>20 \%$, we conducted ROC calculations to establish cut-off values for shoulder/arm disability and psychological distress scores as predictors of significant NDI (\%). AUC analysis for the condition "NDI (\%) significant (yes/no)" showed the following results: Quick-DASH, $0.89 \pm 0.018$ (95\% CI, 


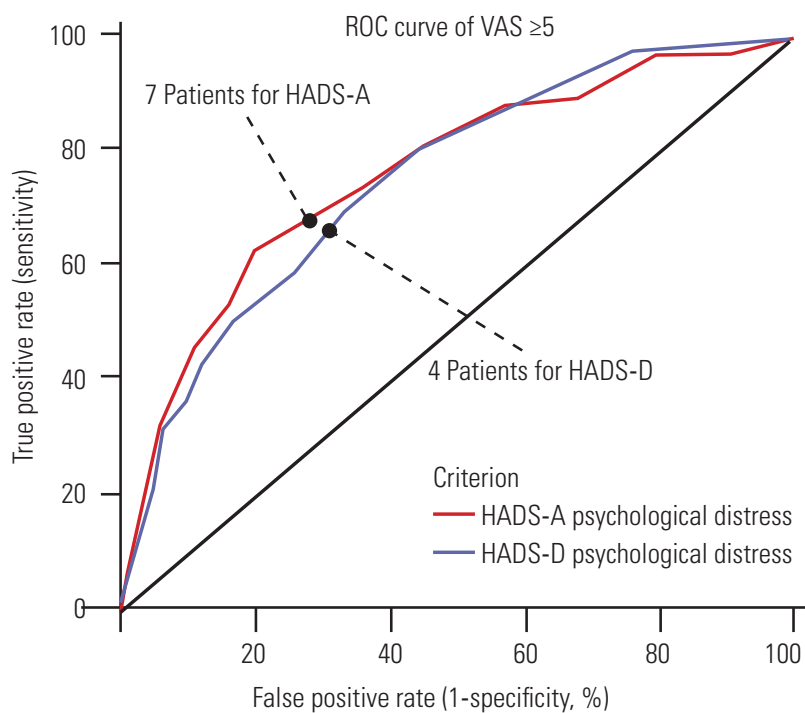

Fig. 6. ROC curve for prediction analysis of VAS-neck/VAS-arm $\geq 5$. The first plot shows the empirical ROC curve. The second plot shows a ROC curve based on the binormal assumption. The ROC shows that both the HADS-A and HADS$D$ were predictive for VAS $\geq 5$ with a cut-off at seven patients related to a sensitivity of $68 \%$ and a specificity of $73 \%$ for the HADS-A and a cut-off of 4 patients related to a sensitivity of $69 \%$ and a specificity of $67 \%$ for the HADSD. ROC, receiver operation characteristic; VAS, Visual Analog Scale; HADS-A, Hospital Anxiety and Depression Scale-anxiety disorders; HADS-D, Hospital Anxiety and Depression Scale-depression.

0.85-0,93), DASH-work, $0.82 \pm 0.023$ (95\% CI, 0.77-0.86); HADS-D score, $0.80 \pm 0.020$ (95\% CI, 0.76-0,84); VASarm, $0.78 \pm 0.025$ (95\% CI, 0.73-0.83); and HADS-A score, $0.77 \pm 0.024$ (95\% CI, 0.72-0.81). Comparisons of the discrimination power according to differences in AUC showed that the difference between the arm of VAS score and HADS-A score ( $p=0.81)$, HADS-D score $(p=0.6)$, and DASH-work $(p=0.1)$ were not significant. AUC of VASarm was significantly smaller than that of Quick-DASH $(p<0.0001)$. The differences between the HADS-A and HADS-D scores were not significant. Differences between Quick-DASH and HADS-A score $(p<0.0001)$ and QuickDASH and HADS-D score $(p=0.0002)$ were significant in favor of Quick-DASH. ROC calculations and curves are shown in Fig. 5 for best cut-offs points sensitivity and specificity values are provided. Total $6 \%$ of the patients reported a neck VAS score of $\geq 5$ in the previous 4 weeks, and $45 \%$ reported an arm VAS score $\geq 5$.

The prevalence of arm and/or neck pain with a VAS score $\geq 5$ within 4 weeks was $7.5 \%$ in the study sample. It was more likely in women $(p=0.01)$ and highest in the age group of 70-90 years where $15 \%$ of subjects reported a VAS score of $\geq 5$, followed by those aged $40-50$ years $(9 \%)$ and those aged $50-60$ years $(10 \%)$. The significant $(p<0.01)$ age-dependent distribution is shown in Supplemental Fig. 1. To study the predictors of arm and neck VAS scores $\geq 5$, AUC-analyses were performed again and a significant impact of psychological distress scores was noted. Discrimination power according to differences of AUC were as follows: HADS-A score $0.76 \pm 0.29$ (95\% CI, 0.70-0.82); HADS-D score $0.73 \pm 0.03$ (95\% CI, 0.67-0.79). The differences were not significant $(p=0.25)$. The ROC calculations were performed for cut-offs as per the sensitivity/specificity levels. The ROC-plot selection is illustrated in Fig. 6.

Regarding psychological distress parameters, $25 \%$ of the participants showed a clinically significant HADS score of $>7$ points. (HADS-A or D scores). The HADS scores were significantly higher in women $(p<0.05)$ but were not dependent on age. The HADS scores correlated with neck and shoulder disability variables $(p<0.05)$ (Table 2 , Fig. $3)$; however, the correlations between measures of neck (NDI\%, neck VAS score) and shoulder disability (mConstant score, arm VAS score, Quick-DASH) were stronger $(p<0.0001, r=0.5$ to $r=0.6)$.

Correlations between parameters for neck disability, shoulder-disability, BMI, and psychological distress in perspective of varying clinical characteristics, such as sex and smokers, are summarized in Supplemental Table 1.

\section{Generic scores and questions}

A summary of the results from the cross-sectional assessment of 1,000 individuals showed that $<1 \%$ of the participants had frequent/continuous limitations of social, private, or occupational activities because of neck pain, and $20 \%$ reported rare limitations. In our study, $18 \%$ of the participants complained about restrictions at work because of neck pain, and $28 \%$ reported difficulties in sleeping. Restrictions in AODL, sleep, and at work were significantly associated with measures of neck and shoulder disability and psychological distress (each $p<0.01$ ). Limited neck rotation was more common in participants who were older, were psychologically distressed, and had neck and shoulder disability (each $p<0.001$ ). Total $9 \%$ of the subjects reported a limitation in neck rotation.

The duration of hand numbness and finger tingling (33\%) was highest among smokers $(p=0.007)$. Moreover, subjects with an increased NDI\% and BMI were more likely to experience these problems ( $p<0.0001$ and $p=0.03$, respectively). Analysis of age-related differences showed the highest prevalence in those aged 50-60 years old (43\%, 
$p<0.001)$. Age-related distribution is shown in Supplemental Fig. 2. Total $6.5 \%$ of all participants reported difficulties to "pick up and grip things." Numbness, weakness, or tingling in the lower extremities was reported by $12 \%$ of participants.

Total $28 \%$ of all patients complained about occasional headaches, judged as "coming from the neck." There was a statistically significant difference between men (16\%) and women $(39 \%)(p<0.0001)$. In subjects with occasional headaches, there were stronger correlations between the measures of neck disability and psychological distress. In general, female sex had a negative influence on the severity of disability measures (arm VAS score $p<0.001$, neck VAS score $p<0.001$, NDI\% $p<0.001$, and HADS scores $p<0.001)$.

Total 34\% of the participants were smokers. Smokers had an increased likelihood of neck and shoulder disability (NDI, trend at $p=0.08$; mConstant score, $p=0.02$ ) as well as reduced neck rotation (trend at $p=0.08$ ). Moreover, the strength of correlation between neck and shoulder disability as well as neck disability and psychological distress measures were stressed in smokers (Supplemental Table 1).

We found that $5 \%$ of the participants had a BMI $\geq 35$ $\mathrm{kg} / \mathrm{m}^{2}$, indicating obesity, and $19 \%$ had a BMI $\geq 30 \mathrm{~kg} /$ $\mathrm{m}^{2}$, indicating overweight. Only a BMI $\geq 35 \mathrm{~kg} / \mathrm{m}^{2}$ showed significant correlation with shoulder-disability (QuickDASH, mConstant score, $p<0.05)$ and psychological distress measures (HADS score, $p<0.01$ ). In contrast, BMI did not correlate with the arm VAS score, neck VAS score, or NDI\% ( $p=0.17, p=0.14$, and $p=0.20$, respectively).

The need for medical care utilization and pain medication increased while neck rotation decreased with higher neck and shoulder disability and psychological distress (each $p<0.001$ ). Total $8 \%$ of the participants reported a need for oral pain medication due to neck pain within the previous 4 weeks. We found that $12 \%$ women and 5\% men required regular pain medication for neck pain; the sex-based difference was significant $(p<0.0001)$. Moreover, higher HADS-A scores were significantly linked with an elevated intake of painkillers (5\% patients had HADS-A score $\leq 7$ points, and $38 \%$ had a score $>7$ points; $p<0.0001)$.

With respect to occupation, unemployment $(n=45)$ was linked with an increased incidence and severity of neck and shoulder disability and psychological distress (arm VAS score, $p=0.04$; neck VAS score, $p=0.01$; NDI\%, $p=0.02$; HADS-D score, $p=0.01$; HADS-A score trend,
Table 4. Summary table of clinical variables linked to a statistically increased probability (risk factor analysis) for increased NDI in a normal population of 1,000 Caucasians

Clinical variables linked to increased NDI

Shoulder and arm problems

Psychological distress levels

Patient age

Female gender

Occupation

Obesity

Dysphagia

Smoking

For statistical strength of interdependencies and other correlations with NDI see the manuscript, Table 3, and Fig. 5.

NDI, Neck Disability Index.

$p=0.08$; and Quick-DASH, $p=0.01)$. Measures of neck and shoulder disability $(p<0.001)$ were highest in nurses and lowest in soldiers. Employees showed an overall higher morbidity than established merchants and manual workers. The occupation-related differences are illustrated in Supplemental Fig. 3.

Regarding dysphagia, $6 \%$ of the participants reported swallowing difficulties for liquids (rarely, sometimes, or often) and $9 \%$ had swallowing difficulties for solids (rarely, sometimes, or often). Problems with swallowing liquids were more common in subjects with higher neck VAS score $(p<0.0001)$ and NDI $(p<0.0001)$, while there was no link with age $(p=0.22)$. Problems in swallowing solids were also significantly correlated with neck VAS score, NDI, HADS-A score, HADS-D score (each $p<0.0001$ ) and age $(p=0.04)$. A general linear discriminant analysis showed that for the category "significant swallowing difficulty," defined as dysphagia reported for liquids or solids, in descending order the NDI\% (Wilk's lambda $=0.986$, $p=0.001$ ) and HADS-D score (Wilk's lambda $=0.987$ ) had the most discrimination power. The total correct classification rate was $90 \%$ in the learning sample and $92 \%$ in the cross-validation group, indicating that the model performs well and the results are stable in the learning and test samples.

A brief and summarizing overview of clinically most relevant correlations between NDI and variables tested is given in Table 4. 


\section{Discussion}

The current study provides considerable valuable information based on a cross-sectional assessment of 1,000 individuals regarding the prevalence of neck and shoulder disability as well as psychological distress. The raw data support a better interpretation of patient-reported disability patterns and study reports regarding outcome measures. The study data also enable the matching of patients in the cervical spine treatment-related outcome studies with otherwise "normal" individuals in future studies. Outcomes of cervical surgery can be normalized to our results. A comparison to "normal" can provide a better clinical perspective. Accordingly, a large sample with normative data for neck, shoulder, and psychological distress is now available as the control group. In clinical practice, the data can help the physician interpret the severity of patient symptoms in regard to that in the general population.

An analysis of the correlations between neck and shoulder disability measures and psychological distress emphasizes the strong interdependence in perceiving and reporting disabilities of the neck and shoulder as well as psychological well-being. Our data are expected to increase the awareness of physicians and surgeons of specific sub-specialties, such as spine surgeons and shoulder surgeons, during the analysis of symptoms and functions of the patient when they present with a neck or a shoulder disorder. It is noteworthy that $45 \%$ of the subjects with significant neck pain ( $\geq 5$ points) also reported shoulder and arm problems. Results might indicate a behavioral problem; therefore, this information can be useful for understanding pain reporting and pain patterns in patients who seek specific orthopedic therapy, such as that for the neck or the shoulder. Furthermore, our data stressed that perception of neck and shoulder disability as well as reporting is significantly elevated in based on the patient's demographic characteristics, sec, occupation, psychological distress levels (HADS score). In a normal population, patient-related risk factors for elevated neck and shoulder disability measures included female sex, smoking, obesity, and older age. A clear link between higher neck disability in terms of significant NDI and psychological distress scores could be identified with cut-offs established for the HADS-D score at 7 points. Such data warrant careful evaluation of shoulder function and related disability reporting and psychological environment analysis in patients with the above-mentioned risk factors in clinical practice. A better understanding of the incidences and interdependencies of neck and shoulder disability as well as psychological distress can improve decision-making and patient counseling with respect to symptoms, outcomes, and expectations.

Although our data were assessed in a large number of patients under defined conditions, the generalizability of our results is of interest. One study by Kato et al. [8] on the normative values for the Japanese NDI version reported a normal NDI of $10.6 \pm 15.2$ in 1,200 subjects, while Bunketorp et al. [15] reported a normal NDI of 7.0 \pm 9.6 in 1,491 subjects; both studies reporting significantly higher NDI values in women than in men. In the current study, NDI was $7.25 \pm 9.26$, and the results correspond well to previous reports. In addition, Kato et al. [8] found that those aged 50-59 years had the highest NDI; these findings are in keeping with our results (Fig. 1). Fejer et al. [16] found a 1-month prevalence of about $25 \%$ for neck pain. In our study sample, $21 \%$ of the participants reported on some limitation in their daily activities because of neck pain. Our data were collected in a hospital setting where higher stress levels might be present and confound the data; therefore, we compared our HADS-scores to the validation of HADS [13]: in our study sample we found a HADS-A score of $4.9 \pm 3.7$ and a HADS-D score of 3.2 \pm 3.5 , comparing favorably to the published normal values for a population with a mean HADS-A score of 5.0 \pm 3.4 and a mean HADS-D score of $4.7 \pm 3.9$ [17]. The findings add further credence to the generalizability of our data.

In our sample, women had significantly different arm VAS score, neck VAS score, NDI\%, HADS-A, and HADSD. Total $27.5 \%$ of our study subjects complained of headaches. Here, we found a significant difference between women $(39 \%)$ and men $(17 \%)(p<0.0001)$. These results are consistent with a study where the prevalence of most common forms of pain was higher among women than among men [18]. Multiple biological and psychosocial processes were contributing factors. Studies show that sex hormones influence pain sensitivity. Psychosocial processes, such as pain coping and early-life exposure to stress, may also explain the sex-based differences in pain, in addition to the role played by sex in pain expression $[3,19]$.

Interpretation of interdependencies between neck and shoulder disability and psychological distress is difficult in a cross-sectional study because it is difficult to define 
the causative indicator variable. In a similar manner, Mäntyselka et al. [20] found that in 604 subjects without depressive symptoms at baseline, at the 7-year follow-up, frequent neck and shoulder pain was a preceding symptom for depressive symptomatology. In his review on the psychological risk factors in back and neck pain, Linton [6] reported strong evidence for a clear link of psychological factors and the onset of neck pain and the transition from acute to chronic pain disability. Similarly, he found that depression, anxiety, distress, and related emotions were associated with pain and disability. He concluded that psychosocial factors might be used as predictors for the development of long-term pain and disability. In our study sample, $89 \%$ of the patients had a HADS-D $<7$ points, and $77 \%$ had HADS-A $<7$ points. It is noteworthy that the HADS groups were significantly different in terms of arm VAS score, neck VAS score, and NDI (all $p<0.0001$ ).

Arm/hand numbness is a commonly evaluated clinical parameter that is assessed in patients with cervical stenosis, given that is believed to be a clinical sign of cervical myelopathy. In this context, it is noteworthy that 33\% of all subjects complained about a period of numbness in their arms/hands within a 4 -week period. The incidence increased with the severity of the NDI $(p<0.0001)$. Moreover, smokers had a higher incidence of numbness in the arms and hands (39\% versus 30\%, $p=0.007$ ). Results emphasize that "numbness" in arms/hands should be interpreted carefully in coalescence of other indices for cervical myelopathy.

The employment type and status had an impact on neck and shoulder disability $(p<0.001)$; disability was highest among unemployed subjects and nurses and lowest among soldiers. Employees showed an overall higher morbidity than established merchants or manual workers. High job demands, high levels of distress, workplacebullying, organizational justice, and limited control over their work situation have already been associated with an elevated incidence of neck pain $[2,4,5]$; this might explain the significantly higher disability in nurses in our study sample (Supplemental Fig. 3).

Dysphagia is a significant perioperative outcome and benchmark criterion in cervical surgery. In the current study, subjects reported a $6 \%-9 \%$ rate of dysphagia. The prevalence in this study is interesting, with $12 \%-14 \%$ of patients reporting persistent dysphagia one year after anterior cervical spine surgery [21]. The prevalence of dysphagia in our sample was increased in individuals with higher neck disability and psychological distress. Data echo early clinical observations [22] and are of interest because most studies report on surgery-related risk factors for postoperative dysphagia, such as previous surgery, multilevel surgery, use of bone morphogenetic protein, and others $[23,24]$. The current data might facilitate the interpretation of elevated postoperative dysphagia rates in at-risk patient subgroups in future surgical studies.

Limitations of our study are related to the lack of imaging data, such as magnetic resonance imaging data, and correlation analysis of imaging results, such as degenerative scores with clinical scores. Future studies on this subject are warranted.

\section{Conclusions}

A prospective, survey-based assessment was performed on 1,000 subjects for the reconstruction of normative values of neck (NDI, VAS-neck) and shoulder disability (Quick-DASH, mConstant score, VAS-arm) and related psychological distress. The average NDI\% was 7.3, and $11 \%$ of the subjects reported a significant NDI, defined as NDI $>20 \%$. Patient-related risk factors for increased neck disability included female sex, age, higher BMI, occupational status, smoking, higher shoulder disability, and psychological distress.

Neck and shoulder disability as well as psychological distress scores were strongly correlated. Results after treatment of cervical spine pathologies can be normalized to the data provided and put into a better perspective of normalcy. Better understanding of the normal interdependencies of neck and shoulder disability and psychological distress enable superior patient counseling.

\section{Conflict of Interest}

No potential conflict of interest relevant to this article was reported.

\section{Author Contributions}

Conception and design: H. Koller; acquisition of data: J. Koller, S. Krebs, M. Mayer, C. Bismark; drafting the article: J. Koller, H. Koller, W. Hitzl; reviewed submitted version of manuscript: all authors; critically revising the article: H. Koller; and approved the final version of the manuscript on behalf of all authors: H. Koller, J. Koller. 


\section{Supplementary Materials}

Supplementary materials can be available from https:/doi. org/10.31616/asj.2019.0397. Supplemental Table 1. Correlation analysis of continuous variables. Supplemental Fig. 1. Age dependent distribution of patients with VAS-arm and/or VAS-neck $\geq 5$ patients. Supplemental Fig. 2. Age dependent distribution of patients that report a period of numbness in arms \& hands in the last 4 weeks prior to survey assessment. Supplemental Fig. 3. Distribution of neck disability and psychological distress according to occupational status of 1,000 subjects.

\section{References}

1. Kaaria S, Laaksonen M, Rahkonen O, Lahelma E, Leino-Arjas P. Risk factors of chronic neck pain: a prospective study among middle-aged employees. Eur J Pain 2012;16:911-20.

2. McLean SM, May S, Klaber-Moffett J, Sharp DM, Gardiner E. Risk factors for the onset of non-specific neck pain: a systematic review. J Epidemiol Community Health 2010;64:565-72.

3. Ramirez-Maestre C, Esteve R. The role of sex/gender in the experience of pain: resilience, fear, and acceptance as central variables in the adjustment of men and women with chronic pain. J Pain 2014;15:60818.

4. Saastamoinen P, Laaksonen M, Leino-Arjas P, Lahelma E. Psychosocial risk factors of pain among employees. Eur J Pain 2009;13:102-8.

5. Cote P, van der Velde G, Cassidy JD, et al. The burden and determinants of neck pain in workers: results of the Bone and Joint Decade 2000-2010 Task Force on Neck Pain and Its Associated Disorders. Spine (Phila Pa 1976) 2008;33(4 Suppl):S60-74.

6. Linton SJ. A review of psychological risk factors in back and neck pain. Spine (Phila Pa 1976) 2000;25:1148-56.

7. Peolsson A, Peolsson M. Predictive factors for longterm outcome of anterior cervical decompression and fusion: a multivariate data analysis. Eur Spine J 2008;17:406-14.

8. Kato S, Takeshita K, Matsudaira K, Tonosu J, Hara N, Chikuda H. Normative score and cut-off value of the Neck Disability Index. J Orthop Sci 2012;17:687-93.

9. Radcliff KE, Koyonos L, Clyde C, et al. What is the incidence of dysphagia after posterior cervical sur- gery? Spine (Phila Pa 1976) 2013;38:1082-8.

10. Vernon H, Mior S. The Neck Disability Index: a study of reliability and validity. J Manipulative Physiol Ther 1991;14:409-15.

11. London DA, Stepan JG, Boyer MI, Calfee RP. Performance characteristics of the verbal QuickDASH. J Hand Surg Am 2014;39:100-7.

12. Boehm D, Wollmerstedt N, Doesch M, Handwerker M, Mehling E, Gohlke F. Development of a questionnaire based on the Constant-Murley-Score for self-evaluation of shoulder function by patients. Unfallchirurg 2004;107:397-402.

13. Herrmann-Lingen C, Buss U, Snaith RP. HADS-D: Hospital Anxiety and Depression Scale: Deutsche version. Bern: Huber; 2011.

14. Bazaz R, Lee MJ, Yoo JU. Incidence of dysphagia after anterior cervical spine surgery: a prospective study. Spine (Phila Pa 1976) 2002;27:2453-8.

15. Bunketorp L, Stener-Victorin E, Carlsson J. Neck pain and disability following motor vehicle accidents: a cohort study. Eur Spine J 2005;14:84-9.

16. Fejer R, Kyvik KO, Hartvigsen J. The prevalence of neck pain in the world population: a systematic critical review of the literature. Eur Spine J 2006;15:83448.

17. Hinz A, Schwarz R. Anxiety and depression in the general population: normal values in the Hospital Anxiety and Depression Scale. Psychother Psychosom Med Psychol 2001;51:193-200.

18. Fillingim RB, King CD, Ribeiro-Dasilva MC, RahimWilliams B, Riley JL 3rd. Sex, gender, and pain: a review of recent clinical and experimental findings. J Pain 2009;10:447-85.

19. Bartley EJ, Fillingim RB. Sex differences in pain: a brief review of clinical and experimental findings. $\mathrm{Br}$ J Anaesth 2013;111:52-8.

20. Mantyselka P, Lupsakko T, Kautiainen H, Vanhala M. Neck-shoulder pain and depressive symptoms: a cohort study with a 7-year follow-up. Eur J Pain 2010;14:189-93.

21. Cho SK, Lu Y, Lee DH. Dysphagia following anterior cervical spinal surgery: a systematic review. Bone Joint J 2013;95-B:868-73.

22. Kang SS, Lee JS, Shin JK, Lee JM, Youn BH. The association between psychiatric factors and the development of chronic dysphagia after anterior cervical spine surgery. Eur Spine J 2014;23:1694-8. 
23. Lu DC, Tumialan LM, Chou D. Multilevel anterior cervical discectomy and fusion with and without rhBMP-2: a comparison of dysphagia rates and outcomes in 150 patients. J Neurosurg Spine 2013;18:439.
24. Smith-Hammond CA, New KC, Pietrobon R, Curtis DJ, Scharver CH, Turner DA. Prospective analysis of incidence and risk factors of dysphagia in spine surgery patients: comparison of anterior cervical, posterior cervical, and lumbar procedures. Spine (Phila $\mathrm{Pa}$ 1976) 2004;29:1441-6. 